\title{
On Simplifying the Function of Many Variable Computing Problems with Symmetry

\author{
Yong Huang
}

College of Mathematics and Statistics, Zhaotong College, Zhaotong Yunnan, 657000, China

Keywords: Symmetry, Function of many variables, Multiple integrals, Curve integral, Surface integral.

Abstract. Symmetry is of important significance in mathematics and more extended region and it can simplify calculus calculation. In this paper we systematically explore of function of many variables Computing Problems by symmetry.

\section{Introduction}

Definite integral of symmetrical simplified univariate function can also be simplified to integral of multivariate function. Integrals of multivariate function include multiple integral and line $\&$ surface integral. The calculation of line and surface integral is the calculation converting to multiple integral, thus, the integral of multivariate function is the calculation of multivariate function. This article will make systematic discussion by using the symmetry and anti-symmetry multivariate function integral issue.

The essence of function symmetry is about the symmetry of geometric figure or geometry, which includes the symmetry about point, straight line and plane of geometric figure or geometry. Common relations between symmetry and anti-symmetry of univariate function $y=f(x)$ about points and straight line are as below:

Function $f(x)$ symmetric with respect to the point $(a, b) \Leftrightarrow f(a+x)+f(a-x)=2 b$

$$
\Leftrightarrow f(x)=f(2 a-x)-2 b
$$

Function $f(x)$ symmetric with respect to $x=a($ anti- symmetric)

$$
\begin{array}{r}
\Leftrightarrow f(a+x)=f(a-x) \quad(f(a+x)=-f(a-x)) \\
\Leftrightarrow f(x)=f(2 a-x) \quad(f(x)=-f(2 a-x))
\end{array}
$$

Thus obtain $\int_{a-x}^{a+x} f(t) d t=2 \int_{0}^{a+x} f(t) d t \quad\left(\int_{a-x}^{a+x} f(t) d t=0\right)$

Similar conclusion for multivariate function can also be obtained. But conditions of symmetry for multivariate function are complicated. We hereby mainly discuss some particular conditions of multivariate function:

\section{Preliminary knowledge}

Definition 1 Assume function $f(x, y)$ defined in region $\mathrm{D},(a, b) \in D$, if $\forall(x, y) \in D$, obtain $(2 a-x, 2 b-y) \in D$, and $f(x, y)=-f(2 a-x, 2 b-y)$, thus call $f(x, y)$ symmetric with respect to the point $(a, b, 0)$ in $\mathrm{D}$.

Definition 2 Assume function $f(x, y)$ defined in region $\mathrm{D}$, if $\forall(x, y) \in D$, thus $(-x, y) \in D$, and $f(-x, y)=f(x, y) \quad(f(-x, y)=-f(x, y))$, then call $f(x, y)$ symmetric with respect to the plan $x=0$ in $\mathrm{D}$ (anti-symmetric ) 
Definition 3 Assume function $f(x, y)$ defined in region $\mathrm{D}$, if $\forall(x, y) \in D$, thus $(2 a-x, y) \in D$, and $f(x, y)=f(2 a-x, y)(f(x, y)=-f(2 a-x, y))$, then call $f(x, y)$ symmetric with respect to the plan $x=a$ in $\mathrm{D}$ (anti- symmetric)

Definition 4 Assume function $f(x, y)$ defined in region $\mathrm{D}$, if $\forall(x, y) \in D$, thus $(-x,-y) \in D$, and $f(-x,-y)=f(x, y) \quad(f(-x,-y)=-f(x, y))$, thus call $f(x, y)$ symmetric with respect to the $Z$ axis (origin anti- symmetric)

\section{Relevant theorem and deduction}

\section{Double integral problem}

theorem 1 If function $f(x, y)$ symmetric with respect to the plane $x=0$ in domain of integration D (anti-symmetric), thus $\iint_{D} f(x, y) d x d y=2 \iint_{D_{1}} f(x, y) d x d y \quad\left(\iint_{D} f(x, y) d x d y=0\right)$

Herein $D_{1}=\{(x, y) \mid(x, y) \in D, x \geq 0\}$

Prove: order $D_{2}=D \backslash D_{1}=\{(x, y) \mid(x, y) \in D, x<0\}$

$$
\iint_{D} f(x, y) d x d y=\iint_{D_{1}} f(x, y) d x d y+\iint_{D_{2}} f(x, y) d x d y
$$

Therein the second form for variable substitution: $x=-u, y=v . \quad D \rightarrow D^{\prime} . \quad J(u . v)=-1$

$$
\iint_{D_{2}} f(x, y) d x d y=\iint_{D^{\prime}} f(-u, v)|J(u, v)| d u d v=\iint_{D^{\prime}} f(-u, v) d u d v=\iint_{D_{1}} f(-x, y) d x d y
$$

Thus $\iint_{D} f(x, y) d x d y=\iint_{D_{1}} f(x, y) d x d y+\iint_{D_{1}} f(-x, y) d x d y=\iint_{D_{1}}[f(x, y)+f(-x, y)] d x d y$

i.e.

when $f(x, y)$ symmetric with respect to $x=0$ in domain of integration $\mathrm{D}$, thus $\iint_{D} f(x, y) d x d y=2 \iint_{D_{1}} f(x, y) d x d y$

when $f(x, y)$ anti-symmetric with respect to $x=0$ in domain of integration $\mathrm{D}$, hence $\iint_{D} f(x, y) d x d y=0$

theorem 2 If function $f(x, y)$ symmetric with respect to the plane $y=0$ in domain of integration $\mathrm{D}$ (anti-symmetric), then $\iint_{D} f(x, y) d x d y=2 \iint_{D_{1}} f(x, y) d x d y \quad\left(\iint_{D} f(x, y) d x d y=0\right)$

Therein $D_{1}=\{(x, y) \mid(x, y) \in D, y \geq 0\}$

theorem 3 If function $f(x, y)$ symmetric with respect to the plane $x=0$ and $y=0$ in domain of integration $\mathrm{D}$, then

$$
\left.\iint_{D} f(x, y) d x d y=4 \iint_{D_{1}} f(x, y) d x d y \quad \iint_{D} f(x, y) d x d y=0\right)
$$

Therein $D_{1}=\{(x, y) \mid(x, y) \in D, x \geq 0, y \geq 0\}$ 
Deduction 1 If function $f(x, y)$ symmetric with respect to the plane $x=a$ in domain of integration $\mathrm{D}$ (anti-symmetric), thus $\iint_{D_{1}} f(x, y) d x d y=\iint_{D_{2}} f(2 a-x, y) d x d y \quad\left(\iint_{D} f(x, y) d x d y=0\right)$. Therein $D_{1}, D_{2}$ symmetric with respect to $x=a$ with no common interior points, $D=D_{1} \cup D_{2}$.

Deduction 2 If function $f(x, y)$ symmetric with respect to the axis $\left\{\begin{array}{l}x=a \\ y=b\end{array}\right.$ in domain of integration $\mathrm{D}$, then $\iint_{D_{1}} f(x, y) d x d y=\iint_{D_{2}} f(2 a-x, y) d x d y=\iint_{D_{3}} f(x, 2 b-y) d x d y=\iint_{D_{4}} f(2 a-x, 2 b-y) d x d y$ therein $D_{1}, D_{2}, D_{3}, D_{4}$ symmetric with respect to straight lines $x=a, y=b$ in $\mathrm{x}$ and y plane with no common interior points, $D=D_{1} \cup D_{2} \cup D_{3} \cup D_{4}$

Deduction 3 If function $f(x, y)$ symmetric with respect to the point $(a, b, 0)$ in domain of D, then $\iint_{D} f(x, y) d x d y=0$

Deduction 4 If function $f(x, y)$ symmetric with respect to the plane $y=x$ in domain of D,then $\iint_{D} f(x, y) d x d y=\iint_{D} f(y, x) d x d y$.

Similarly, $f(z, x) f(y, z)$ symmetric with respect to planes parallel to coordinate planes, straight lines parallel to coordinate axis and points in coordinate planes. Hence obtain relevant conclusion.

\section{Triple integral problem}

For ternary function $f(x, y, z)$, symmetry of integrand is not easy to be explained from geometrics, but above conclusion can still be promoted. Common conclusions are as below:

Theorem 4 Assume $f(x, y, z)$ defined in integral domain $\mathrm{G}: \quad z=\varphi(x, y)$, thus

( i ) $\forall(x, y, z) \in G,(x, y,-z) \in G$, and $f(x, y,-z)=f(x, y, z) \quad(f(x, y,-z)=f(x, y, z))$ then $\iiint_{G} f(x, y, z) d x d y d z=2 \iiint_{G_{1}} f(x, y, z) d x d y d z \quad\left(\iiint_{G} f(x, y, z) d x d y d z=0\right)$

( ii ) $\forall(x, y, z) \in G,(-x,-y, z) \in G$, 且 $f(-x,-y, z)=f(x, y, z)(f(-x,-y, z)=-f(x, y, z))$ 则 $\iiint_{G} f(x, y, z) d x d y d z=2 \iiint_{G_{1}} f(x, y, z) d x d y d z \quad\left(\iiint_{G} f(x, y, z) d x d y d z=0\right)$

(iii ) $\forall(x, y, z) \in G,(-x,-y,-z) \in G, f(-x,-y,-z)=f(x, y, z) \quad(f(-x,-y,-z)=-f(x, y, z))$ 则 $\iiint_{G} f(x, y, z) d x d y d z=2 \iiint_{G_{1}} f(x, y, z) d x d y d z \quad\left(\iiint_{G} f(x, y, z) d x d y d z=0\right)$

Deduction $5 \quad \forall(x, y, z) \in G \quad, \quad(-x,-y,-z) \in G \quad, \quad f(-x,-y,-z)=f(x, y, z)$ $(f(-x,-y,-z)=-f(x, y, z))$, and when domain $\mathrm{G}$ symmetric with respect to plane $x y, y z, z x$,obtain $\iiint_{G} f(x, y, z) d x d y d z=8 \iiint_{G_{1}} f(x, y, z) d x d y d z \quad\left(\iiint_{G} f(x, y, z) d x d y d z=0\right)$

Therein $G_{1}=\{(x, y, z) \mid(x, y, z) \in G, x \geq 0, y \geq 0, z \geq 0\}$

\section{Curvilinear integral problem}

For functions defined in planes or space curve sections, the symmetry is also not easy to be explained from geometrics, but above conclusion can still be promoted. Common conclusions are as below: 
Theorem 5 Assume $f(x, y)$ continuous in flat section smooth curve L, if curve symmetric with respect to $x$, and $f(x,-y)=f(x, y) \quad(f(x,-y)=-f(x, y))$ thus $\int_{L} f(x, y) d s=2 \int_{L_{1}} f(x, y) d s$ $\left(\int_{L} f(x, y) d s=0\right)$

Prove: assume $L=L^{\prime}+L^{\prime \prime}$ is smooth curve symmetric with respect to $x$ axis, $L^{\prime}, L^{\prime \prime}$ are located in two sides of $x$ axis respectively.

Partition: any component curve $L^{\prime}$ is n segmental arc $L_{i}^{\prime} \quad(i=1,2, \cdots, n)$, accordingly, $L^{\prime \prime}$ is divided into n segmental arc $L_{i}^{\prime \prime}$ symmetrically. Arc length of $L_{i}^{\prime}, L_{i}^{\prime \prime}$ are $\Delta S_{i}^{\prime}, \Delta S_{i}^{\prime \prime}$, record $\Delta S_{i}=\Delta S_{i}^{\prime}=\Delta S_{i}^{\prime \prime}$. Fineness of partition is $\|T\|=\max _{1 \leq i \leq n}\left\{\Delta S_{i}\right\}$.

Access point: $\forall P\left(\xi_{i}, \eta_{i}\right) \in L_{i}^{\prime}$, accordingly, its symmetric point $P^{\prime} \in\left(\xi_{i},-\eta_{i}\right) \in L_{i}^{\prime \prime}$, therein $L^{\prime}, L^{\prime \prime}$ symmetric with respect to $x$ axis.

Summation: $\sum_{i=1}^{n} f\left(\xi_{i}, \eta_{i}\right) \Delta S_{i}+\sum_{i=1}^{n} f\left(\xi_{i},-\eta_{i}\right) \Delta S_{i}$

Take limit: $\lim _{\|T\| \rightarrow 0} \sum_{i=1}^{n} f\left(\xi_{i}, \eta_{i}\right) \Delta S_{i}+\lim _{\|T\| \rightarrow 0} \sum_{i=1}^{n} f\left(\xi_{i},-\eta_{i}\right) \Delta S_{i}$

When $f\left(\xi_{i},-\eta_{i}\right)=f\left(\xi_{i}, \eta_{i}\right)$, its limit is $2 \lim _{\|T\| \rightarrow 0} \sum_{i=1}^{n} f\left(\xi_{i}, \eta_{i}\right) \Delta S_{i}$

i.e. $\int_{L} f(x, y) d s=2 \int_{L_{1}} f(x, y) d s$

when $f\left(\xi_{i},-\eta_{i}\right)=-f\left(\xi_{i}, \eta_{i}\right)$, its limit is 0 ,i.e. $\int_{L} f(x, y) d s=0$

Deduction 6 Assume function $f(x, y)$ continuous in flat section smooth curve, if curve symmetric with respect to both $\mathrm{x}$ and $\mathrm{y}$ axis, then

( i ) when $f(-x, y)=f(x,-y)=f(x, y)$, obtain $\int_{L} f(x, y) d s=4 \int_{L_{1}} f(x, y) d s$

( ii ) when $f(-x, y)=-f(x, y)$ or $f(x,-y)=-f(x, y)$, obtain $\int_{L} f(x, y) d s=0$

\section{Surface integral problem}

Theorem 6 Assume function $f(x, y, z)$ continuous in spatial lamination smooth curved surface. If S symmetric with respect to both $\mathrm{x}$ and $\mathrm{y}$ plane, and $f(x, y,-z)=f(x, y, z)$ $\left(f(x, y,-z)=-f(x, y, z)\right.$ thus $\iint_{S} f(x, y, z) d s=2 \iint_{S_{1}} f(x, y, z) d s \quad\left(\iint_{S} f(x, y, z) d s=0\right)$

Therein $S_{1}$ is an unilateral part of $x y$ plane

Deduction 7 Assume $f(x, y, z)$ continuous in piecewise smooth curved surface S. If curve symmetric with respect to $x y, y z, z x$ planes, thus

( i ) when $f(-x, y, z)=f(x,-y, z)=f(x, y,-z)=f(x, y, z)$,

$$
\iint_{S} f(x, y, z) d s=8 \iint_{S_{1}} f(x, y, z) d s
$$

( ii ) when $f(-x, y, z)=-f(x, y, z)$ or $f(x,-y, z)=-f(x, y, z)$ or $f(x, y,-z)=-f(x, y, z)$, obtain $\iint_{S} f(x, y, z) d s=0$

Therein $S_{1}$ is a part of the first octant. 


\section{Problems need attention for symmetry of function}

The essence of function symmetry is about the symmetry of geometric figure or geometry image of two-variable functions is the curve of three-dimensional space.

Double integral $\iint_{D} f(x, y) d x d y$ refers to the volume of curved-roof cylinder with the top of $f(x, y)$ in geometrics. For ternary function $f(x, y, z)$ and functions defined in planes or space curve sections or special curved surfaces, their symmetry is not easy to be explained in geometrics. Relevant conclusions of their symmetry are mainly obtained by deducting from symmetric conclusions of two-variable functions. Their integral values are related to integrand symmetry but also related with the symmetry of geometrical shape in integral region.

\section{As the integral curve (plane) of type two curve (plane) is directing curve (constant lateral).}

Its value is related with the geometrical shape of integrand and integral curve (plane), but also related with the side of integral curved surface. When using symmetry, except for considering the symmetry in two aspects: geometrical shape of integrand and integral curve (plane), we should also consider normal direction pointing of integral curve (plane). Thus when calculating the integral of type two curve (plane), we should consider whether we can use its symmetry to simplify the calculation after converting the integral of type two curve (plane) to the integral of type one curve (plane) or double integral.

\section{Acknowledgments}

This paper is supported by the scientific research foundation of Yunnan Provincial Department of Education (2014Y499).

\section{References}

[1] Department of mathematics, East China Normal University, First edition. Beijing: Higher Education Press.

[2] Huang Yong, Kang Daokun. Studies on symmetric simplified univariate function integral computation problems, Journal of Longdong University, 2011(4), 1-3.

[3] Feng Aiping. Studies on symmetry of multivariate function, Journal of Xi'an University of Science and Technology, 2007 (3): 527-530.

[4] Wang Xianjie. Calculation of double integral and triple integral in symmetric domain, Journal of Mudanjiang normal university, 2007 (4): 65-66.

[5] Liu Weichaun. Use symmetry to calculate curvilinear integral and surface integral, Henan Science. 2006, 24 (6): 810-812.

[6] Liu Fugui. Methods of using symmetry to calculate the second curvilinear integral and surface integral, Journal of Wuhan University of Technology,2006, 12 (6):1069-1072.

[7] Chen Yunxin. Application of symmetry of functional displacement in calculating integral, Studies In College Mathematics, 2001, 4 ()12: 8-31. 